\title{
QOS Challenges for Next Generation Mobile Middleware
}

\author{
Andrew T. Campbell \\ Department of Electrical Engineering and \\ Center for Telecommunications Research \\ Columbia University, New York, NY 10027-6699 \\ http://comet.ctr.columbia.edu/ campbell \\ http://comet.ctr.columbia.edu/wireless
}

\section{INTRODUCTION}

The development of next generation mobile multimedia communications systems [2] presents a number of technical challenges which are thus far unresolved. First, it is essential that QOS assurances be given for the transfer of audio and video flows to mobile devices as they migrate between cells in cellular systems. Second, future mobile communications systems must be able to provide dynamic re-routing of a set of multimedia flows associated with a mobile device from one base station to another in a timely manner, without significantly interrupting the flows in progress and with a smooth change in the quality delivered. Third, existing multimedia transport systems are ineffective when operating in environments where widespread mobility and changing network characteristics are dominant. These challenges, due primarily to large-scale mobility requirements, limited radio resources and fluctuating network conditions, fundamentally impact our ability to deliver multimedia flows over mobile and, in general, QOS fluctuating networks.

In this position statement we present a number of QOS challenges for next generation mobile middleware. Specifically, we address the need for QOS-aware middleware for mobile multimedia communications and illustrate one possible approach being developed in the wireless media systems [6] project at Columbia University. For an extended version of this position statements see the web [7].

\section{QOS CHALLENGES}

A number of key questions need to be resolved when investigating QOS-aware middleware. First, what is the relationship between the middleware layer and the wireline and wireless network? The radio channel's varying QOS characteristics and device mobility fundamentally impact our ability to deliver hard QOS guarantees in the wireless environment. Therefore, what is the level of QOS (i.e., service class) which can be ensured by the network to the middleware platform (e.g., hard, soft, or no guarantees)? Based on this result, what is a suitable QOS abstraction to offer to mobile multimedia applications? 
Second, what is the impact of handoff on mobile multimedia flows? A flow with certain capacity reserved at a particular cell may have to be re-routed when the mobile device changes its location. The new path to the desired location may not have the original required capacity. Therefore, re-negotiation of resources allocated to the connection is needed. At the same time, the flow (e.g., audio or video) should be transported and presented 'seamlessly' to the destination device with a smooth change of perceptual quality.

Next, what is the ability of the mobile multimedia applications to adapt when resources fluctuate over time. This is dependent on the type of flow. For example, continuous media flows have built-in redundancy where media scaling algorithms can exploit the intrinsic scalable properties of multi-resolution audio and video flows when the delivered QOS fluctuates.

Fourth, audio and video flows are characterized by the production, transmission and consumption of single media streams (both unicast or multicast) with associated QOS. For multicast flows, individual receivers (both wired and wireless) may have differing capabilities to consume flows. This could be due to either fluctuating network resources with mobility or imposed by individual applications. How do we bridge this heterogeneity gap in mobile multicast environments while simultaneously meeting the individual mobile devices' QOS requirements?

In order to clarify these issues and put them into the proper perspective, we present a brief description of one platform which addresses these QOS challenges. The mobiware platform sets the stage for a brief discussion of the type of new architecture and algorithms needed to realize next generation mobile middleware

\section{MOBIWARE: QOS-AWARE MOBILE MIDDLEWARE}

Mobiware is a software middleware platform that runs seamlessly on mobile devices, base stations and mobile-capable ATM switches based on xbind [3] programmability. As illustrated in figure 1, mobiware operates in the region between the radio and wired ATM communications firmware, and mobile multimedia applications. The goal of QOSaware middleware is to provide a highly programmable platform for delivering continuous media flows in mobile multimedia networks. The concepts of programmability [3] and adaptability [1] are central to managing the complexity of delivering voice, video and data with high quality over QOS-varying mobile networks during handoff and periods of persistent QOS fluctuation. The mobiware platform is built on xbind (built itself on CORBA technology) and Java distributed systems technology and incorporates new architecture and novel adaptive algorithms to support the concept of 'QOS controlled mobility'.

Mobiware provides mobile multimedia applications with a new QOS abstraction called QOS controlled mobility. This involves scaling flows during handoff rather than dropping them when desired resources are unavailable. The benefits of such an approach is to reduce handoff dropping and improve wireless resource utilization. We use the term "controlled QOS" to distinguish it from hard QOS guarantees offered by fixed ATM networks. Implicit in the term is the notion that flows can be represented and transported as multi-layer scalable flows at the mobile device as illustrated in figure 2. Adaptive algorithms help scale flows during handoff based on available bandwidth and an 
application-specific 'flow adaptation policy' [1]. This policy characterizes each audio and video flows as having a minimum QOS layer and a number of enhancements.

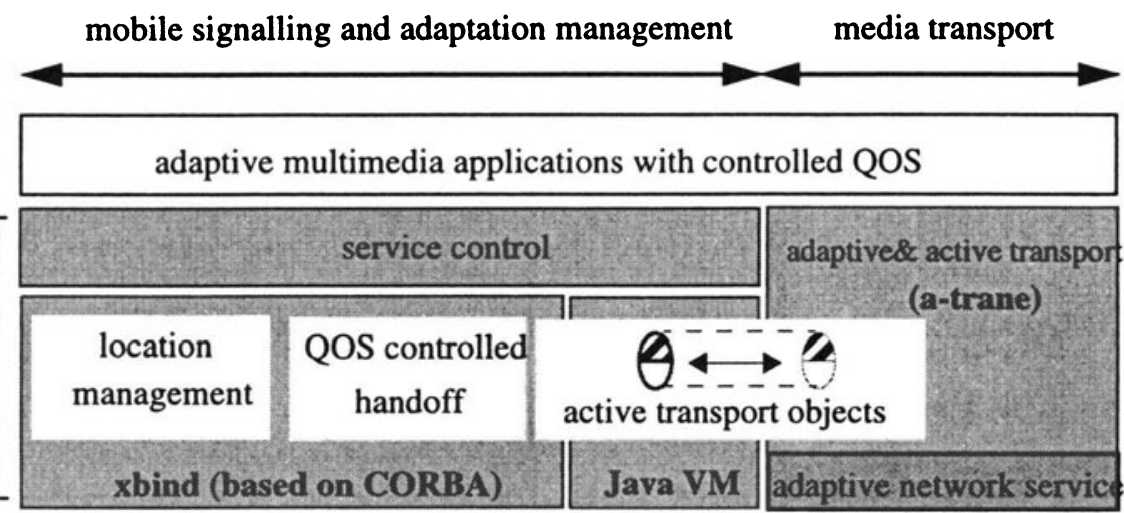

wireline/wireless network

Figure 1: Mobiware

Mobiware is built on three major algorithmic components: First, QOS controlled mobility, is based on the seamless delivery of media through interaction with an adaptive network service, media scaling objects, and an adaptive and active transport system called $a$-trane; the handoff algorithm employes advanced algorithms which model flows using mobile soft-state, aggregate flows using the concept of connection groups and limit the impact of small scale mobility of the wired network through the introduction of local anchor points for routing and adaptation - for full details of the handoff algorithm see [5];

Next, an adaptive network service, characterizes flows as having a base layer (BL) and a number of enhancement layers (viz. E1 and E2). The base layer provides a foundation for better resolutions to be delivered through the reception of enhancement layers based on the availability of resources in the wireless environment - for full details on the adaptive network service which offers hard guarantees to the BL and uses QOS adaptation for enhancement layers see [1]; and

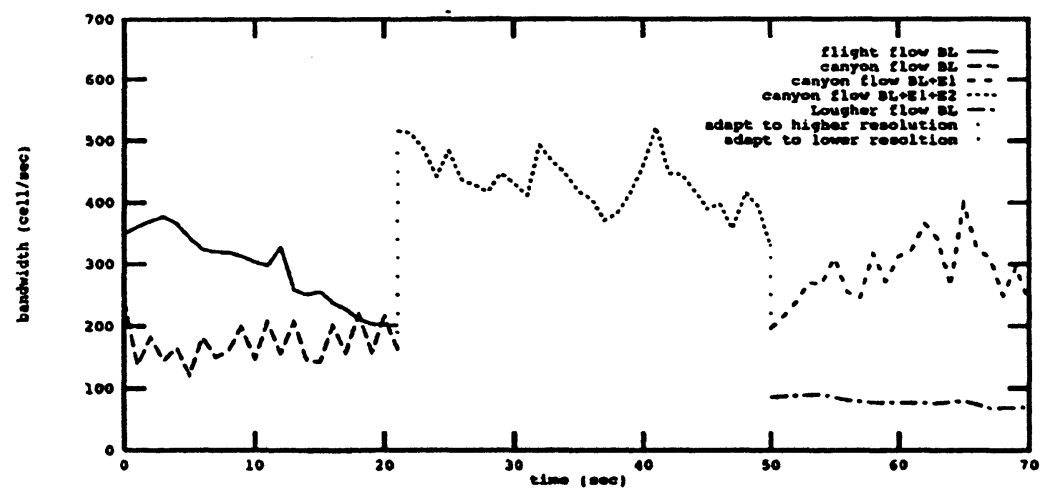

Figure 2: QOS Controlled Mobility 
Finally, adaptive and active transport called a-trane, supports the transfer of multi-layer flows through the provision of a QOS-based API and a set of dynamically loadable active transport objects (ATOs) (e.g., active filters [4] and error control objects [1]). Dynamically loadable transport objects can be dispatched to the base station and mobile devices to support valued-added QOS when and where it is needed e.g., during periods of QOS fluctuations and degradation. Currently mobiware supports active filters and error control ATOs - for full details on a-trane see [6].

\section{REFERENCES}

[1] Campbell, A., "Mobiware: QOS-aware Middleware for Mobile Multimedia Communications", Proc. IFIP 7th Intl. Conf. on High Performance Networking, White Plains, New York, April 1997.

[2] Raychaudhuri, D., et al., "Scope and Work Plan for Proposed Wireless ATM Working Group", ATM Forum Technical Committee, ATM Forum/96-0530/ PLEN, April, 1996.

[3] Lazar, A.A., Bhonsle, S. and Lim, K.S., "A Binding Architecture for Multimedia Networks", Journal of Parallel and Distributed Computing, Vol. 30, Number 2, November 1995, pp. 204-216.

[4] Balachandran A., Campbell A.T. and Kounavis M., "Active Filters: Delivering Scaled Media to Mobile Devices", Proc. 7th International Workshop on Network and Operating System Support for Digital Audio and Video, St Louis, May 1997.

[5] Campbell, A.T., Liao, R-F. and Shobatake Y., "Supporting QOS Controlled Handoff in Mobiware", 6th Winlab Workshop on 3rd Generation Wireless Information Networks, New Brunswick, March 20-21, 1997.

[6] comet.ctr.columbia.edu/wireless: Wireless Media Systems Project at Columbia University.

[7] Campbell, A. T., QOS Challenges for Next Generation Mobile Middleware (Extended Version), comet.ctr.columbia.edu/wireless/publications 